\title{
TOTALLY GEODESIC HOMEOMORPHISMS BETWEEN TEICHMÜLLER SPACES
}

\author{
Dong Tan \\ Guangxi University, College of Mathematics and Information Science \\ 530000, Guangxi, P. R. China; duzuizhe2013@foxmail.com
}

\begin{abstract}
First, we show that a projective measured foliation is a Busemann point, in Gardiner-Masur boundary, if and only if it is indecomposable. Let $f: \mathcal{T}_{g, n} \rightarrow \mathcal{T}_{g, n}$ be a totally geodesic homeomorphism and suppose that $f$ admits a homeomorphic extension to $\partial_{\mathrm{GM}} \mathcal{T}_{g, n}$. We show that $f$ induces a simplicial automorphism of curve complex. Moreover, the restriction of $f$ on $\mathcal{T}_{g, n}$ is an isometry. As an application, we obtain an alternative proof of Royden's Theorem.
\end{abstract}

\section{Introduction}

Let $S=S_{g, n}$ be a Riemann surface of genus $g$ with $n$ punctures. Denote by $\mathcal{T}_{g, n}$ the Teichmüller space of $S$ and $d_{\mathcal{T}}(\cdot, \cdot)$ the Teichmüller metric.

Much of the study of the geometry on Teichmüller space is inspired by analogies with hyperbolic space. When $S$ is a flat torus or a punctured torus, the Teichmüller metric is isometric to $\mathbf{H}^{2}$. When $3 g-3+n \geq 2$, Masur [18] proved that the Teichmüller metric does not have negative curvature in the sense of Busemann. Masur and Wolf [19] proved that the Teichmüller metric is not Gromov hyperbolic. However, there have been various successful attempts to generalize the geometry of $\mathbf{H}^{2}$ to Teichmüller spaces of higher dimension $[9,12]$.

A map from a geodesic metric space to itself is called totally geodesic if it maps any geodesic to a geodesic. Jeffers [8] showed that:

Theorem 1. Let $\mathbf{H}^{n}$ denote the hyperbolic space of dimension $n$. Suppose that $f: \mathbf{H}^{n} \rightarrow \mathbf{H}^{n}(n \geq 2)$ is a totally geodesic bijection. Then $f$ is an isometry.

$\mathrm{Li}$ and Wang [14] even showed that either the injective or surjective condition of $f$ is unnecessary.

Motivated by the above works, Lixin Liu suggested the following conjecture:

Conjecture 1.1. Let $f$ be a totally geodesic homeomorphism from the Teichmüller space $\mathcal{T}_{g, n}$ to itself, with respect to the Teichmüller metric. Then $f$ is an isometry.

In this paper we make some progresses for the above conjecture.

1.1. Main results. Throughout this paper, we assume that $3 g-3+n \geq 2$ and endow $\mathcal{T}_{g, n}$ with the Teichmüller metric. Let $\widehat{\mathcal{T}}_{g, n}$ denote the Gardiner-Masur compactification of Teichmüller space, and $\operatorname{Mod}_{g, n}^{ \pm}$be the extended mapping class group of $S$, i.e. the group of isotopy classes of diffeomorphisms of $S$.

Our main results are given as follows:

https://doi.org/10.5186/aasfm.2020.4538

2010 Mathematics Subject Classification: Primary 32G15, 30F60, 57M50. geodesic.

Key words: Gardiner-Masur boundary, mapping class group, Teichmüller space, totally

The work is partially supported by NSFC No. 11771456 
Theorem 2. Let $f: \mathcal{T}_{g, n} \rightarrow \mathcal{T}_{g, n}$ be a totally geodesic homeomorphism and suppose that $f$ admits a homeomorphic extension to $\partial_{\mathrm{GM}} \mathcal{T}_{g, n}$. Then $f$ is induced by an element of the mapping class group $\operatorname{Mod}_{g, n}^{ \pm}$, except $(g, n)=(1,2)$. In particular, $f$ is an isometry.

Remark 1.2. When $S=S_{g, n}$ is a torus with two punctures, i.e. $(g, n)=(1,2)$, we will show that $f \in \operatorname{Mod}_{0,5}^{ \pm}$, which is the isometry group of $\mathcal{T}_{1,2}$ and the automorphism group of the complex of curves on $S_{1,2}$.

The importance of Theorem 2 is to give a new analogies between the geometry of Teichmüller space and that of the hyperbolic space. The proof of Theorem 2 is inspired by Ivanov's proof of Royden's Theorem [7]. Note that the next two theorems (Theorem 1.3 and Theorem 1.4) play important roles during the proof of Theorem 2.

Recall that the Gardiner-Masur boundary is the boundary of the GardinerMasur compactification of $\mathcal{T}_{g, n}$ [2]. Moreover, Gardiner and Masur in [2] showed that the Gardiner-Masur boundary contains the Thurston boundary $\mathcal{P} \mathcal{M} \mathcal{F}$, the space of projective measured foliations on $S$ (cf. Section 2.2).

By a result of $\mathrm{Liu}-\mathrm{Su}$ [16], any isometry of $\mathcal{T}_{g, n}$ admits a homeomorphic extension to $\widehat{\mathcal{T}}_{g, n}$. As an application, Theorem 2 also gives a new proof of Royden's theorem:

Theorem 3. (Royden) The isometry group of $\mathcal{T}_{g, n}$ endowed with the Teichmüller metric is $\operatorname{Mod}_{g, n}^{ \pm}$, except $(g, n)=(1,2)$ or $(2,0)$.

Let $\mathcal{M F}$ denote the space of measured foliations on $S$. A measured foliation is indecomposable if it has only one indecomposable component. We refer to Subsection 2.2 for the definition of indecomposable components of measured foliations.

Let $X \in \mathcal{T}_{g, n}$ and $[\mathcal{F}] \in \mathcal{P} \mathcal{M F}$. Denote by $\mathbf{R}_{X, \mathcal{F}}:[1, \infty) \rightarrow \mathcal{T}_{g, n}$ the Teichmüller geodesic ray associated to $[\mathcal{F}]$ emanating from $X$ (cf. Section 2.3). In [16], Liu and $\mathrm{Su}$ proved that every Teichmüller geodesic ray converges to a unique point on the Gardiner-Masur boundary. Denote by $\mathcal{B}_{X}(\mathcal{F}):=\lim _{K \rightarrow \infty} \mathbf{R}_{X, \mathcal{F}}(K)$ the limit of Teichmüller geodesic ray in Gardiner-Masur boundary. By applying some results of Walsh in [27], we generalize a result of Miyachi [20] as follows.

Theorem 1.3. For a measured foliation $\mathcal{F}$, the limit $\mathcal{B}_{X}(\mathcal{F})$ is equal to the projective class of some measured foliation if and only if $\mathcal{F}$ is indecomposable.

The curve complex $C(S)$ of a surface $S$ was introduced into the study of Teichmüller space by Harvey [3] as an analogue of the Tits building of a symmetric space. The vertices of Harvey's complex are homotopy classes of simple closed curves in $S$, and the simplices are collections of curves that can be realized disjointly.

Let $\mathcal{S}$ denote the set of homotopy classes of non-trivial and non-peripheral simple closed curves on $S$. Then we establish

Theorem 1.4. Let $f: \mathcal{T}_{g, n} \rightarrow \mathcal{T}_{g, n}$ be a totally geodesic homeomorphism and suppose that $f$ admits a homeomorphic extension to $\partial_{\mathrm{GM}} \mathcal{T}_{g, n}$. Then, the following hold:

(1) The map $f$ acts homeomorphically on $\mathcal{P} \mathcal{M F} \subset \partial_{\mathrm{GM}} \mathcal{T}_{g, n}$.

(2) The restriction of $f$ to $\mathcal{P} \mathcal{M F}$ preserves $\mathcal{S}$ (i.e. $f(\mathcal{S})=\mathcal{S}$ ) and induces a simplicial automorphism of the complex of curves.

1.2. Organization of the article. In $\S 2$, we give the preliminaries on Teichmüller theory and measured foliations. In §3, we introduce the Gardiner-Masur boundary and prove that a projective measured foliation in $\mathcal{P} \mathcal{M F}$ is a Busemann point if and only if it is "indecomposable". The proof relies on recent work of Walsh 
[26]. In $\S 4$, we use the intersection relationship between measured foliations to show that $f$ induces an automorphism of the complex of curves. Theorem 2 is proved in $\S 5$.

Acknowledgements. The authors are grateful to Lixin Liu and Weixu Su for their helpful suggestions and discussions.

\section{Prelaminaries}

In this section, we recall some basic notions in Teichmüller theory and measured foliations.

2.1. Teichmüller space. Let $S$ be a Riemann surface of genus $g$ with $n$ punctures, with $3 g-3+n \geq 2$. The Teichmüller space $\mathcal{T}_{g, n}$ is the space of equivalence classes of pairs $(X, f)$, where $X$ is a Riemann surface of analytically finite type $(g, n)$ and $f: S \rightarrow X$ is a diffeomorphism (known as a marking). The equivalence relation is given by $\left(X_{1}, f_{1}\right) \sim\left(X_{2}, f_{2}\right)$ if there is a bi-holomorphic mapping $\phi: X_{1} \rightarrow X_{2}$ so that $\phi \circ f_{1}$ is isotopic to $f_{2}$. Denote the equivalent class of $(X, f)$ by $[(X, f)]$.

The Teichmüller space $\mathcal{T}_{g, n}$ has a complete metric called the Teichmüller metric. For any $\left[\left(X_{1}, f_{1}\right)\right],\left[\left(X_{2}, f_{2}\right)\right] \in \mathcal{T}_{g, n}$ the distance function of the Teichmüller metric is defined by

$$
d_{\mathcal{T}}\left(\left[\left(X_{1}, f_{1}\right)\right],\left[\left(X_{2}, f_{2}\right)\right]\right)=\frac{1}{2} \inf _{h} \log K(h),
$$

where $h$ ranges over all quasiconformal mappings $h: X_{1} \rightarrow X_{2}$ homotopic to $f_{2}^{-1} \circ f_{1}$, and $K(h)$ is the maximal quasiconformal dilatation of $h$.

For simplicity, we shall denote a point in $\mathcal{T}_{g, n}$ by a Riemann surface $X$, without explicit reference to the marking or to the equivalence relation.

2.2. Measured foliations. A measured foliation $\mathcal{F}$ on $S$ is a foliation (with a finite number of singularities) with a transverse invariant measure. At each singularity $p$ including punctures, there exists $k \geq 1$ such that the singularity is locally modelled on a $k$-pronged singularity of $z^{k-2} d z^{2}$, where $k \geq 3$ if $p$ is not a puncture. A leaf of $\mathcal{F}$ is called critical if it contains a singularity of $\mathcal{F}$. The union of the compact critical leaves is called the critical graph.

A measured foliation $\mathcal{F}$ on $S$ is said to be minimal if all leaves are either dense in $S$ or join two singularities - and there must be no cycle of leaves of the latter type. A measured foliation $\mathcal{F}$ is called uniquely ergodic if the support of $\mathcal{F}$ is minimal and admits a unique transverse measure. A measured foliation $\mathcal{F} \in \mathcal{M F}$ is rational if any non-critical trajectory is closed. It is clear that any rational measured foliation is identified with a formal sum of mutually disjoint weighted simple closed curves.

The intersection number $i(\gamma, \mathcal{F})$ of a simple closed curve $\gamma$ with a measured foliation $\mathcal{F}$ endowed with transverse measure $\mu$ is defined by

$$
i(\gamma, \mathcal{F})=\inf _{\gamma^{\prime}} \int_{\gamma^{\prime}} d \mu
$$

where the infimum is taken over all simple closed curves $\gamma^{\prime}$ in the isotopy class of $\gamma$.

Two non-zero measured foliations $\mathcal{F}$ and $\mathcal{F}^{\prime}$ are measure equivalent if, for all $\gamma \in \mathcal{S}, i(\gamma, \mathcal{F})=i\left(\gamma, \mathcal{F}^{\prime}\right)$. Denote by $\mathcal{M F}=\mathcal{M} \mathcal{F}(S)$ the space of equivalence classes of measured foliations on $S$.

Two measured foliations $\mathcal{F}$ and $\mathcal{F}^{\prime}$ are projectively equivalent if there is a constant $b>0$ such that $\mathcal{F}=b \cdot \mathcal{F}^{\prime}$, i.e. $i(\gamma, \mathcal{F})=b \cdot i\left(\gamma, \mathcal{F}^{\prime}\right)$ for all $\gamma \in \mathcal{S}$. The space of projective equivalence classes of foliations is denoted by $\mathcal{P} \mathcal{M} \mathcal{F}$. 
Thurston showed that $\mathcal{M F}$ is homeomorphic to a $6 g-6+2 n$ dimensional ball and $\mathcal{P} \mathcal{M F}$ is homeomorphic to a $6 g-7+2 n$ dimensional sphere. For more details on measured foliations see [1].

We will use the ergodic decomposition of a measured foliation later in this paper. By removing the critical graph, a measured foliation $\mathcal{F}$ is decomposed into a finite number of connected components, each of which is either a cylinder of closed leaves or a minimal component in which every leaf is dense in its support subsurface. Furthermore, the transverse measure on a minimal component $D$ may be written as a sum of a finite number of projectively distinct ergodic measures:

$$
\left.\mu\right|_{D}=\sum_{k} \mu_{D, k}
$$

We refer to $[5,11]$ for more details.

Definition 2.1. A measured foliation $\mathcal{F}^{\prime}$ is an indecomposable component of $\mathcal{F}$ if it is either one of the cylindrical components of $\mathcal{F}$, or it is measure equivalent to one of the minimal components $D \subset S$ with one of the transverse measures $\mu_{D, k}$.

Definition 2.2. A measured foliation $\mathcal{F}$ is indecomposable if it has only one indecomposable component, i.e. it is equivalent to either a weighted simple closed curve or a minimal component on a subsurface with an ergodic measure.

We denote the set of indecomposable measured foliations on $S$ by $\mathcal{M F}_{\text {ind }}$. We can represent any measured foliation $\mathcal{F}$ as a finite sum

$$
\mathcal{F}=\sum_{i=1}^{k} \mathcal{F}_{i}
$$

of mutually non-intersecting $\left(i\left(\mathcal{F}_{i}, \mathcal{F}_{j}\right)=0\right)$ indecomposable measured foliations. In the literature, such a (unique) decomposition is called the ergodic decomposition of $\mathcal{F}$.

The next lemma will be used later.

Lemma 2.3. [26, Lemma 6.3] Let $\left\{\mathcal{F}_{i}\right\}_{i=0}^{n}$ be a set of projectively-distinct indecomposable elements of $\mathcal{M \mathcal { F }}$ such that $i\left(\mathcal{F}_{i}, \mathcal{F}_{j}\right)=0$ for all $i$ and $j$. Then for any $\varepsilon>0$, there exists a simple closed curve $\beta \in \mathcal{S}$ such that

$$
i\left(\mathcal{F}_{i}, \beta\right)<i\left(\mathcal{F}_{0}, \beta\right) \varepsilon, \forall i \neq 0 \text {. }
$$

2.3. Quadratic differentials. A holomorphic quadratic differential $q$ on $X \in$ $\mathcal{T}_{g, n}$ is a tensor locally represented by $q=q(z) d z^{2}$, where $q(z)$ is a holomorphic function under the local coordinate $z=x+i y$. We allow holomorphic quadratic differentials to have simple poles at the punctures of $X$. The cotangent space of $\mathcal{T}_{g, n}$ at $X$ can be identified with the vector space $\mathcal{Q}(X)$ of holomorphic quadratic differentials on $X$. The $L^{1}$-norm on $\mathcal{Q}(X)$ is defined by

$$
\|q\|=\int_{X}|q|
$$

It is the dual norm for defining the Teichmüller metric.

A quadratic differential $q \in \mathcal{Q}(X)$ gives rise to a pair of transverse measured foliations $\mathcal{F}_{v}(q)$ and $\mathcal{F}_{h}(q)$ on $X$, called the vertical and horizontal measured foliation of $q$, respectively. In natural coordinates such that $q=d z^{2}, \mathcal{F}_{v}(q)\left(\mathcal{F}_{h}(q)\right)$ is defined by the leaves $x=$ constant $(y=$ constant) with the measure $|d x|(|d y|)$. 
According to a fundamental theorem of Hubbard and Masur [4], for any $\mathcal{F} \in \mathcal{M F}$ and $X \in \mathcal{T}_{g, n}$, there is a unique holomorphic quadratic differential $q \in \mathcal{Q}(X)$ such that $\mathcal{F}_{v}(q)$ is measured equivalent to $\mathcal{F}$. We call $q$ the Hubbard-Masur differential of $\mathcal{F}$ on $X$.

Let $X \in \mathcal{T}_{g, n}$ and $\mathcal{F} \in \mathcal{M F}$. Let $q$ be the Hubbard-Masur differential of $\mathcal{F}$ on $X$. The Teichmüller geodesic ray $\mathbf{R}_{X, \mathcal{F}}:[0, \infty) \rightarrow \mathcal{T}_{g, n}$ is defined as a family of Riemann surfaces $X_{t}=\mathbf{R}_{X, \mathcal{F}}(t)$ such that each $X_{t}$ is the quasiconformal deformation of $X$ with Beltrami differential

$$
\frac{K-1}{K+1} \frac{|q|}{q}
$$

where $K=e^{2 t}$. The parameter $t$ is chosen such that the Teichmüller distance between $X_{s}$ and $X_{t}$ is equal to $|s-t|$.

2.4. Extremal length. Let $X=[(X, f)] \in \mathcal{T}_{g, n}$. The extremal length $\operatorname{Ext}_{X}(\alpha)$ of a simple closed curve $\alpha \in \mathcal{S}$ is defined by

$$
\operatorname{Ext}_{X}(\alpha)=\sup _{\rho} \frac{\ell_{\rho}(f(\alpha))^{2}}{\operatorname{Area}(X, \rho)},
$$

where the supremum is taken over all conformal metrics $\rho$ of finite area and $\ell_{\rho}(f(\alpha))$ denotes the geodesic length of $f(\alpha)$ with respect to the metric $\rho$.

Kerckhoff [9] proved that there is a unique continuous extension of the extremal length functions from $\mathcal{S}$ to $\mathcal{M F}$ where

$$
\operatorname{Ext}_{X}(r \cdot \alpha)=r^{2} \operatorname{Ext}_{X}(\alpha), r \in \mathbf{R}_{+} .
$$

The following formula is due to Kerckhoff [9].

Theorem 2.4. Given $X, Y \in \mathcal{T}_{g, n}$, the Teichmüller distance

$$
d_{\mathcal{T}}(X, Y)=\frac{1}{2} \log \sup _{\alpha \in \mathcal{S}}\left(\frac{\operatorname{Ext}_{X}(\alpha)}{\operatorname{Ext}_{Y}(\alpha)}\right) .
$$

2.5. Curve complex and mapping class group. The curve complex of a surface was introduced into the study of Teichmüller space by Harvey [3] as an analogue of the Tits building of a symmetric space. The vertex set of the curve complex $\mathcal{C}(S)$ is given by $\mathcal{S}$. Recall that $\mathcal{S}$ is the set of essential and nonperipheral simple closed curves on $S$, considered up to isotopy. Two vertices $\alpha, \beta \in \mathcal{C}(S)$ are connected by an edge if they have disjoint representations. For any two vertices $\alpha, \beta \in \mathcal{C}(S)$, we define the distance $d_{\mathcal{S}}(\alpha, \beta)$ to be the minimal number of edges connecting $\alpha$ and $\beta$.

The mapping class group $\operatorname{Mod}(S)$ is the group of homotopy classes of orientationpreserving diffeomorphisms $\sigma: S \rightarrow S$. Every mapping class $[\sigma]$ acts on $\mathcal{T}_{g, n}$ by changing the marking:

$$
[(X, f)] \rightarrow\left[\left(X, f \circ \sigma^{-1}\right)\right]
$$

Denote by $\operatorname{Mod}^{ \pm}(S)$ the extended mapping class group, which contains $\operatorname{Mod}(S)$ as a subgroup of index two.

It is clear that $\operatorname{Mod}^{ \pm}(S)$ acts on the curve complex as a group of automorphisms.

Theorem 2.5. (Ivanov) If $S$ is not a sphere with $\leq 4$ punctures, nor a torus with $\leq 2$ punctures, then all automorphisms of $\mathcal{C}(S)$ are given by the elements of $\operatorname{Mod}^{ \pm}(S)$.

This famous theorem is due to Ivanov [6] in the case of surfaces of genus at least two and to Korkmaz [10] in the remaining cases. For the torus with two punctures, 
$\mathcal{C}\left(S_{1,2}\right)$ is isomorphic to $\mathcal{C}\left(S_{0,5}\right)$; and the automorphism group of $\mathcal{C}\left(S_{1,2}\right)$ is $\operatorname{Mod}^{ \pm}\left(S_{0,5}\right)$ instead of $\operatorname{Mod}^{ \pm}\left(S_{1,2}\right)$.

\section{Gardiner-Masur boundary and Busemann points}

In this section we define and study the Gardiner-Masur boundary of $\mathcal{T}_{g, n}$. We show that a projective measured foliation is the limit point of a Teichmüller geodesic ray if and only if the measured foliation is indecomposable. This generalizes the works of Miyachi [21, 22].

3.1. Gardiner-Masur boundary. Denote by $\mathbf{R}_{+}^{\mathcal{S}}$ the space of nonnegative functions on $\mathcal{S}$, endowed with the weak topology. Let $P \mathbf{R}_{+}^{\mathcal{S}}$ be the projective space of $\mathbf{R}_{+}^{\mathcal{S}}$.

By using hyperbolic length functions of $\mathcal{S}$, Thurston defined an embedding of $\mathcal{T}_{g, n}$ into $P \mathbf{R}_{+}^{\mathcal{S}}$. The closure of this embedding is compact, called the Thurston compactification. Boundary of the Thurston compactification can be identified with $\mathcal{P} \mathcal{M F}$, by representing each $[\mathcal{F}] \in \mathcal{P} \mathcal{M F}$ with the projective class of the intersection function $i(\mathcal{F}, \cdot): \mathcal{S} \rightarrow \mathbf{R}_{+}^{\mathcal{S}}$. Thus $\mathcal{P} \mathcal{M F}$ is also called the Thurston boundary of $\mathcal{T}_{g, n}$.

The definition of the Gardiner-Masur compactification is similar. We can define an embedding of $\mathcal{T}_{g, n}$ into $P \mathbf{R}_{+}^{\mathcal{S}}$ by

$$
\Phi: X \mapsto\left[\left(\sqrt{\operatorname{Ext}_{X}(\alpha)}\right)_{\alpha \in \mathcal{S}}\right]
$$

Gardiner and Masur [2] showed that the closure of $\Phi\left(\mathcal{T}_{g, n}\right)$ in $P \mathbf{R}_{+}^{\mathcal{S}}$ is compact. The boundary of $\Phi\left(\mathcal{T}_{g, n}\right)$ is the so called the Gardiner-Masur boundary, denoted by $\partial_{\mathrm{GM}} \mathcal{T}_{g, n}$. It contains the Thurston boundary as a proper subset. Unlike the Thurston boundary, the topology of $\partial_{\mathrm{GM}} \mathcal{T}_{g, n}$ is not clear. However, Miyachi [20] showed that

Theorem 3.1. Any point $P \in \partial_{\mathrm{GM}} \mathcal{T}_{g, n}$ can be represented as a nonnegative continuous function $\mathcal{E}_{P}(\cdot)$ on $\mathcal{M F}$ with the following properties:

(1) $\mathcal{E}_{P}(r \cdot \mathcal{F})=r \mathcal{E}_{P}(\mathcal{F})$ for any $r>0$ and $\mathcal{F} \in \mathcal{M F}$

(2) The assignment $\mathcal{S} \ni \alpha \mapsto \mathcal{E}_{P}(\alpha)$ represents $P$ as a point of $\partial_{\mathrm{GM}} \mathcal{T}_{g, n}$.

Furthermore, the function $\mathcal{E}_{P}(\cdot)$ is unique up to multiplication by a positive constant.

$\mathrm{Liu}$ and $\mathrm{Su}[16]$ proved that

Theorem 3.2. The Gardiner-Masur boundary of $\mathcal{T}_{g, n}$ is the horofunction boundary of the Teichmüller metric.

We refer to $[16,27]$ for the definition of horofunction boundary. An immediate corollary of Theorem 3.2 is that any Teichmüller geodesic ray has a unique limit on the Gardiner-Masur boundary.

A point in the horofunction boundary is called a Busemann point if it is the limit point of an almost geodesic ray [23]. In [27], Walsh showed the Busemann points are exactly the limits of the Teichmüller rays. Hence we can think of Busemann point as the limit point of a geodesic ray on the horofunction boundary .

Let $\mathbf{R}_{X, \mathcal{F}}:[0, \infty) \rightarrow \mathcal{T}_{g, n}$ denote the Teichmüller geodesic ray determined by $X \in \mathcal{T}_{g, n}$ and $\mathcal{F} \in \mathcal{M F}$. Denote the Hubbard-Masur differential of $\mathcal{F}$ on $X$ by $q$. Using Theorem 3.1, we denote the limit of $\mathbf{R}_{X, \mathcal{F}}$ on $\partial_{\mathrm{GM}} \mathcal{T}_{g, n}$ by $\mathcal{E}_{q}(\cdot)$.

With the above notions, we have the following result, proved by Walsh [27]. 
Theorem 3.3. Let $\mathcal{F}=\sum_{i=1}^{k} \mathcal{F}_{i}$ be the ergodic decomposition of $\mathcal{F}$. Then the limit of the Teichmüller geodesic ray $\mathbf{R}_{X, \mathcal{F}}$ is given by

$$
\mathcal{E}_{q}(\cdot)=\left\{\sum_{i=1}^{k} \frac{i\left(\mathcal{F}_{i}, \cdot\right)^{2}}{i\left(\mathcal{F}_{i}, \mathcal{F}_{h}(q)\right)}\right\}^{1 / 2} .
$$

3.2. Characterization of Busemann points in $\mathcal{P} \mathcal{M} \mathcal{F}$. When $3 g-3+n \geq 2$, non-Busemann points do exist in $\partial_{\mathrm{GM}} \mathcal{T}_{g, n}$ [22]. In [20], Miyachi has shown

Theorem 3.4. For a rational measured foliation $\mathcal{G}$, the limit $\mathcal{B}_{X}(\mathcal{G})$ is equal to the projective class of some measured foliation if and only if the support of $\mathcal{G}$ consists of one simple closed curve.

The following theorem generalizes Theorem 3.4.

Theorem 3.5. A projective measured foliation $[\mathcal{F}] \in \mathcal{P} \mathcal{M F}$ is a Busemann point if and only if $\mathcal{F}$ is indecomposable.

Proof. Suppose that $\mathcal{F}$ has more than one ergodic components. Let $\mathcal{F}=\sum_{i=1}^{k} \mathcal{F}_{i}$ be the ergodic decomposition of $\mathcal{F}$ and set

$$
\mathcal{E}_{q}(\cdot)=\left\{\sum_{i=1}^{k} \frac{i\left(\mathcal{F}_{i}, \cdot\right)^{2}}{i\left(\mathcal{F}_{i}, \mathcal{F}_{h}(q)\right)}\right\}^{1 / 2} .
$$

It suffices to prove that $\mathcal{E}_{q}(\cdot) \neq i(\mathcal{G}, \cdot)$ for any $\mathcal{G} \in \mathcal{M F}$.

Suppose to the contrary that $\mathcal{E}_{q}(\cdot)=i(\mathcal{G}, \cdot)$ for some $\mathcal{G} \in \mathcal{M} \mathcal{F}$. Since $\mathcal{E}_{q}(\mathcal{G})=$ $i(\mathcal{G}, \mathcal{G})=0$, by Proposition 5.1 in $[20]$, we have $i(\mathcal{F}, \mathcal{G})=0$. Thus we can write $\mathcal{G}$ as

$$
\mathcal{G}=\sum_{i=1}^{k} b_{i} \mathcal{F}_{i}+\mu
$$

where $\mu \in \mathcal{M F}, b_{i} \geq 0$.

For simplicity, we set

$$
m_{i}:=\frac{1}{i\left(\mathcal{F}_{i}, \mathcal{F}_{h}(q)\right)}
$$

Then

$$
\sum_{i=1}^{k} m_{i} i\left(\mathcal{F}_{i}, \gamma\right)^{2}=\left(\sum_{i=1}^{k} b_{i} i\left(\mathcal{F}_{i}, \gamma\right)+i(\mu, \gamma)\right)^{2}
$$

for all $\gamma \in \mathcal{S}$.

First we observe that $\mu=0$. If not, by Lemma 2.3, for any $\epsilon>0$, there exists $\beta \in \mathcal{S}$ such that $i\left(\mathcal{F}_{i}, \beta\right)<i(\mu, \beta) \epsilon$. It follows from (1) that

$$
i(\mu, \beta)^{2}<\left(\sum_{i} m_{i}\right) i(\mu, \beta)^{2} \epsilon^{2} .
$$

Thus

$$
\left(\sum_{i} m_{i}\right) \epsilon^{2}>1
$$

which is impossible since $\epsilon$ can be arbitrary small. It follows that

$$
\sum_{i=1}^{k} m_{i} i\left(\mathcal{F}_{i}, \gamma\right)^{2}=\left(\sum_{i=1}^{k} b_{i} i\left(\mathcal{F}_{i}, \gamma\right)\right)^{2}
$$


By a similar argument, we can check that $m_{i}=b_{i}^{2}$. Thus we obtain

$$
\sum_{i=1}^{k} b_{i}^{2} i\left(\mathcal{F}_{i}, \gamma\right)^{2}=\left(\sum_{i=1}^{k} b_{i} i\left(\mathcal{F}_{i}, \gamma\right)\right)^{2}, \forall \gamma \in \mathcal{S}
$$

By expanding equation (2), the non-negativeness of the intersection number implies that $b_{i}=0, i=1, \ldots, k$. Hence we obtain

$$
\mathcal{G}=\sum_{i=1}^{k} b_{i} \mathcal{F}_{i}+\mu=0
$$

This leads to a contradiction.

\section{Totally geodesic homeomorphisms}

Throughout this section, we let $\widehat{\mathcal{T}}_{g, n}$ denote the Gardiner-Masur compactification of Teichmüller space, and let $f: \mathcal{T}_{g, n} \rightarrow \mathcal{T}_{g, n}$ be a totally geodesic homeomorphism and suppose that $f$ admits a homeomorphic extension to $\partial_{\mathrm{GM}} \mathcal{T}_{g, n}$.

Denote the set of Busemann points in $\widehat{\mathcal{T}}_{g, n}$ by $\mathcal{B}$. Then $f$ defines a map $f: \mathcal{B} \rightarrow \mathcal{B}$. Using the fact that any two points in $\mathcal{T}_{g, n}$ can be connected by a unique geodesic, one can check that the inverse map $f^{-1}$ is also totally geodesic and $f^{-1}: \mathcal{B} \rightarrow \mathcal{B}$.

\subsection{Uniquely ergodic measured foliations.}

Definition 4.1. Let $P, Q \in \mathcal{B}$. We say $P$ and $Q$ are connected by a Teichmüller geodesic if there exists a complete Teichmüller geodesic $\mathbf{R}(t)$ such that

$$
\lim _{t \rightarrow+\infty} \mathbf{R}(t)=P \quad \text { and } \quad \lim _{t \rightarrow-\infty} \mathbf{R}(t)=Q
$$

Denote the above relation by $P \ominus Q$.

Let $\mathcal{P} \mathcal{M F}^{U E}$ denote the set of uniquely ergodic projective measured foliations.

Lemma 4.2. Let $P=[\mathcal{F}] \in \mathcal{P M F}^{U E}$. Then for any $Q \in \mathcal{B}, Q \neq P$, satisfies $P \ominus Q$.

Proof. We can assume that $Q$ is represented by

$$
\mathcal{E}_{Q}(\cdot)=\left(\sum_{i=1}^{k} m_{i} i\left(\mathcal{G}_{i}, \cdot\right)^{2}\right)^{1 / 2}
$$

Set $b_{i}=m_{i} i\left(\mathcal{G}_{i}, \mathcal{F}\right)>0$. We take

$$
\mathcal{G}=\sum_{i=1}^{k} b_{i} \cdot \mathcal{G}_{i}
$$

There exists a unique quadratic differential $q$ such that

$$
\mathcal{F}_{h}(q)=\mathcal{F} \quad \text { and } \quad \mathcal{F}_{v}(q)=\mathcal{G} .
$$

Denote by $\mathbf{R}(t)$ the Teichmüller geodesic directed by $q$. It follows Theorem 3.3 that as $t \rightarrow+\infty, \mathbf{R}(t)$ converges to

$$
\mathcal{E}_{q}(\cdot)=\left(\sum_{i=1}^{k} \frac{i\left(b_{i} \mathcal{G}_{i}, \cdot\right)^{2}}{i\left(b_{i} \mathcal{G}_{i}, \mathcal{F}\right)}\right)^{1 / 2}=\left(\sum_{i=1}^{k} \frac{b_{i}}{i\left(\mathcal{G}_{i}, \mathcal{F}\right)} i\left(\mathcal{G}_{i}, \cdot\right)^{2}\right)^{1 / 2}=\left(\sum_{i=1}^{k} m_{i} i\left(\mathcal{G}_{i}, \cdot\right)^{2}\right)^{1 / 2}
$$


Thus

$$
\lim _{t \rightarrow+\infty} \mathbf{R}(t)=Q
$$

On the other hand, since $\mathcal{F}$ is uniquely ergodic,

$$
\lim _{t \rightarrow-\infty} \mathbf{R}(t)=[\mathcal{F}]
$$

Thus $\mathbf{R}(t)$ connects $P$ and $Q$.

The converse statement is also true:

Lemma 4.3. Let $P \in \mathcal{B}$ be a Busemann point. If $P \ominus Q$ for all $Q \in \mathcal{B}, Q \neq P$, then $P=[\mathcal{F}] \in \mathcal{P} \mathcal{M F}^{U E}$.

Proof. If $P \in \mathcal{B}$ is not the projective class of any uniquely ergodic measured foliation, then we can represent $P$ as (some $\mathcal{F}=\sum_{i=1}^{k} \mathcal{F}_{i}$ )

$$
\mathcal{E}_{P}(\cdot)=\left(\sum_{i=1}^{k} \frac{i\left(\mathcal{F}_{i}, \cdot\right)^{2}}{i\left(\mathcal{F}_{i}, \mathcal{F}_{h}(q)\right)}\right)^{1 / 2} .
$$

We can always choose $\mathcal{G} \in \mathcal{M} \mathcal{F}_{\text {ind }}$ such that $i(\mathcal{F}, \mathcal{G})=0$ and $[\mathcal{G}] \neq[\mathcal{F}]$. Then $P$ and $[\mathcal{G}]$ could not be connected by any Teichmüller geodesic.

Since the relation $P \ominus Q$ is preserved by $f$, by Lemma 4.2 and Lemma 4.3, we have

$$
f\left(\mathcal{P} \mathcal{M} \mathcal{F}^{U E}\right)=\mathcal{P} \mathcal{M F}^{U E}
$$

By the density of $\mathcal{P} \mathcal{M} \mathcal{F}^{U E}$ in $\mathcal{P} \mathcal{M F}$ and the continuity of $f$, we have

$$
f(\mathcal{P M F})=\mathcal{P} \mathcal{M F}
$$

By Theorem 3.5, we also have

$$
f\left(\mathcal{P} \mathcal{M F}_{\text {ind }}\right)=\mathcal{P} \mathcal{M} \mathcal{F}_{\text {ind }}
$$

Note that $\mathcal{P} \mathcal{M} \mathcal{F}_{\text {ind }}=\mathcal{B} \cap \mathcal{P} \mathcal{M F}$.

4.2. Complex of curves. The set of simple closed curves $\mathcal{S}$ is a subset of $\mathcal{P} \mathcal{M} \mathcal{F}_{\text {ind }}$. We will prove that $f(\mathcal{S})=\mathcal{S}$. Moreover, $f$ will induce an automorphism of $\mathcal{C}(S)$, the complex of curves on $S$.

Definition 4.4. Let $\mathcal{F}, \mathcal{G} \in \mathcal{M} \mathcal{F}_{\text {ind }}$. Define $[\mathcal{F}] \bowtie[\mathcal{G}]$ if there exist two sequences $\left\{X_{n}, \mathcal{F}_{n}\right\},\left\{Y_{n}, \mathcal{G}_{n}\right\} \in \mathcal{T}_{g, n} \times \mathcal{M F}$ such that

- $\lim _{n \rightarrow \infty} \mathcal{F}_{n}=\mathcal{F}$ and $\lim _{n \rightarrow \infty} \mathcal{G}_{n}=\mathcal{G}$,

- for each $n$, the rays $\mathbf{R}_{X_{n}, \mathcal{F}_{n}}$ and $\mathbf{R}_{Y_{n}, \mathcal{G}_{n}}$ converge to the same Busemann point.

Note that $\bowtie$ is not an equivalence relation.

Lemma 4.5. The relation $\bowtie$ on $\mathcal{P} \mathcal{M} \mathcal{F}_{\text {ind }}$ is preserved by $f$.

Proof. Let $[\mu],[\nu] \in \mathcal{P} \mathcal{M F}_{\text {ind }}$ be two indecomposable measured foliations and $[\mu] \bowtie[\nu]$. There exist two sequences $\left\{x_{n}, \mu_{n}\right\},\left\{y_{n}, \nu_{n}\right\} \in \mathcal{T}_{g, n} \times \mathcal{M F}$ satisfying

- $\lim _{n \rightarrow \infty} \mu_{n}=\mu$ and $\lim _{n \rightarrow \infty} \nu_{n}=\nu$,

- for every $n$, the geodesic rays $\mathbf{R}_{x_{n}, \mu_{n}}$ and $\mathbf{R}_{y_{n}, \nu_{n}}$ converge to the same Busemann point.

For the restriction of $f$ to $\mathcal{P} \mathcal{M F}$ is a self-homeomorphism of $\mathcal{P} \mathcal{M F}$, we have

$$
\lim _{n \rightarrow \infty} f\left(\left[\mu_{n}\right]\right)=f([\mu]) \text { and } \lim _{n \rightarrow \infty} f\left(\left[\nu_{n}\right]\right)=f([\nu]) .
$$


At the same time, we have

$$
\mathbf{R}_{f\left(x_{n}\right), f\left[\mu_{n}\right]}=f\left(\mathbf{R}_{x_{n}, \mu_{n}}\right) \quad \text { and } \quad \mathbf{R}_{f\left(y_{n}\right), f\left[\nu_{n}\right]}=f\left(\mathbf{R}_{y_{n}, \nu_{n}}\right),
$$

converge to the same Busemann point.

The proof is completed.

Lemma 4.6. $[\mathcal{F}] \bowtie[\mathcal{G}]$ if and only if $i(\mathcal{F}, \mathcal{G})=0$.

Proof. Suppose $[\mathcal{F}] \bowtie[\mathcal{G}]$. By definition, there exist

$$
\left\{X_{n}, \mathcal{F}_{n}\right\},\left\{Y_{n}, \mathcal{G}_{n}\right\} \in \mathcal{T}_{g, n} \times \mathcal{M F}
$$

such that

$$
\lim _{n \rightarrow \infty} \mathcal{F}_{n}=\mathcal{F}, \quad \lim _{n \rightarrow \infty} \mathcal{G}_{n}=\mathcal{G},
$$

and, $\mathbf{R}_{X_{n}, \mathcal{F}_{n}}$ and $\mathbf{R}_{Y_{n}, \mathcal{G}_{n}}$ converge to the same Busemann point. If $i(\mathcal{F}, \mathcal{G})>0$, then as $n$ is sufficiently large, $i\left(\mathcal{F}_{n}, \mathcal{G}_{n}\right)>0$. This contradicts Theorem 3.3.

For the converse, suppose $i(\mathcal{F}, \mathcal{G})=0$. If $\mathcal{F}=\mathcal{G}$, this lemma follows from the definition of " $\bowtie$ ". Hence we assume $\mathcal{F} \neq \mathcal{G}$. For any $n \in \mathbf{N}_{+}$, we can find two uniquely ergodic measured foliations $\mathcal{F}_{n}, \mathcal{G}_{n}$ satisfying the following conditions:

$$
\left\{\begin{array}{l}
i\left(\mathcal{F}, \mathcal{F}_{n}\right) \leq \frac{1}{n}, \quad i\left(\mathcal{G}, \mathcal{F}_{n}\right) \geq 1 \\
i\left(\mathcal{G}, \mathcal{G}_{n}\right) \leq \frac{1}{n}, \quad i\left(\mathcal{F}, \mathcal{G}_{n}\right) \geq 1
\end{array}\right.
$$

In fact, for any $n \in \mathbf{N}^{+}$, by applying Lemma 2.3 , there are two simple closed curves $\alpha, \beta \in \mathcal{S}$ such that

$$
\frac{i(\mathcal{F}, \alpha)}{i(\mathcal{G}, \alpha)}<\frac{1}{n+1}, \quad \frac{i(\mathcal{G}, \beta)}{i(\mathcal{F}, \beta)}<\frac{1}{n+1}
$$

Moreover, there exist two uniquely ergodic measured foliations $\mathcal{F}_{n}^{\prime}, \mathcal{G}_{n}^{\prime}$ such that

$$
\frac{i\left(\mathcal{F}, \mathcal{F}_{n}^{\prime}\right)}{i\left(\mathcal{G}, \mathcal{F}_{n}^{\prime}\right)}<\frac{1}{n}, \quad \frac{i\left(\mathcal{G}, \mathcal{G}_{n}^{\prime}\right)}{i\left(\mathcal{F}, \mathcal{G}_{n}^{\prime}\right)}<\frac{1}{n},
$$

since the set of uniquely ergodic measured foliations is dense in $\mathcal{M F}$ and the intersection function is continuous. We always can choose two constants $s_{n}$ and $t_{n}$ such that

$$
i\left(\mathcal{G}, s_{n} \mathcal{F}_{n}^{\prime}\right)=1 \quad \text { and } \quad i\left(\mathcal{F}, t_{n} \mathcal{G}_{n}^{\prime}\right)=1
$$

This implies that

$$
i\left(\mathcal{F}, s_{n} \mathcal{F}_{n}^{\prime}\right)<\frac{1}{n} \quad \text { and } \quad i\left(\mathcal{G}, t_{n} \mathcal{G}_{n}^{\prime}\right)<\frac{1}{n} .
$$

Let $\mathcal{F}_{n}=s_{n} \mathcal{F}_{n}^{\prime}$ and $\mathcal{G}_{n}=t_{n} \mathcal{G}_{n}^{\prime}$. Hence, we obtain the inequalities (3).

Let

$$
a_{n}=\frac{i\left(\mathcal{F}, \mathcal{F}_{n}\right)}{i\left(\mathcal{F}, \mathcal{G}_{n}\right)} \quad \text { and } \quad b_{n}=\frac{i\left(\mathcal{G}, \mathcal{G}_{n}\right)}{i\left(\mathcal{G}, \mathcal{F}_{n}\right)}
$$

By (3), we have

$$
\lim _{n \rightarrow \infty} a_{n}=0 \quad \text { and } \quad \lim _{n \rightarrow \infty} b_{n}=0 .
$$

Construct two sequences of measured foliations

$$
\left\{\nu_{n}\right\}=\left\{a_{n} \mathcal{F}+\mathcal{G}\right\} \quad \text { and } \quad\left\{\mu_{n}\right\}=\left\{\mathcal{F}+b_{n} \mathcal{G}\right\} .
$$

It is clear that

$$
\mu_{n} \rightarrow \mathcal{F} \quad \text { and } \quad \nu_{n} \rightarrow \mathcal{G}
$$


Let $q_{n}^{\prime}$ (respectively, $q_{n}^{\prime \prime}$ ) be the quadratic differential such that $\mathcal{F}_{v}\left(q_{n}^{\prime}\right)=\nu_{n}$ and $\mathcal{F}_{h}\left(q_{n}^{\prime}\right)=\mathcal{F}_{n}$ (respectively, $\mathcal{F}_{v}\left(q_{n}^{\prime \prime}\right)=\mu_{n}$ and $\mathcal{F}_{h}\left(q_{n}^{\prime \prime}\right)=\mathcal{G}_{n}$ ). We assume $q_{n}^{\prime} \in \mathcal{Q}\left(X_{n}\right)$ and $q_{n}^{\prime \prime} \in \mathcal{Q}\left(Y_{n}\right)$. Then

$$
\mathbf{R}_{X_{n}, \nu_{n}}(t) \rightarrow\left\{\frac{a_{n}}{i\left(\mathcal{F}, \mathcal{F}_{n}\right)} i(\mathcal{F}, \cdot)^{2}+\frac{1}{i\left(\mathcal{G}, \mathcal{F}_{n}\right)} i(\mathcal{G}, \cdot)^{2}\right\}^{1 / 2}
$$

and

Note that

$$
\mathbf{R}_{Y_{n}, \mu_{n}}(t) \rightarrow\left\{\frac{1}{i\left(\mathcal{F}, \mathcal{G}_{n}\right)} i(\mathcal{F}, \cdot)^{2}+\frac{b_{n}}{i\left(\mathcal{G}, \mathcal{G}_{n}\right)} i(\mathcal{G}, \cdot)^{2}\right\}^{1 / 2}
$$

$$
\frac{a_{n}}{i\left(\mathcal{F}, \mathcal{F}_{n}\right)}=\frac{1}{i\left(\mathcal{F}, \mathcal{G}_{n}\right)}, \quad \frac{1}{i\left(\mathcal{G}, \mathcal{F}_{n}\right)}=\frac{b_{n}}{i\left(\mathcal{G}, \mathcal{G}_{n}\right)} \text {. }
$$

Thus $\mathbf{R}_{X_{n}, \mu_{n}}(t)$ and $\mathbf{R}_{Y_{n}, \nu_{n}}(t)$ converge to the same Busemann point. By the above construction, we know that $[\mathcal{F}] \bowtie[\mathcal{G}]$.

Let $[\mathcal{F}] \in \mathcal{P} \mathcal{M F}_{\text {ind }}$. Denote by

$$
\mathcal{N}([\mathcal{F}])=\left\{[\mathcal{G}] \in \mathcal{P} \mathcal{M} \mathcal{F}_{\text {ind }} \mid i(\mathcal{F}, \mathcal{G})=0\right\} .
$$

The following lemma can be prove by using [6, Theorem 4.1].

Lemma 4.7. Let $[\mathcal{F}],[\mathcal{G}] \in \mathcal{P} \mathcal{M} \mathcal{F}_{\text {ind }}$. Then $\mathcal{N}([\mathcal{F}])=\mathcal{N}([\mathcal{G}])$ if and only if $\mathcal{F}$ and $\mathcal{G}$ are topological equivalent.

Proposition 4.8. Let $f: \mathcal{T}_{g, n} \rightarrow \mathcal{T}_{g, n}$ be a totally geodesic homeomorphism and suppose that $f$ admits a homeomorphic extension to $\partial_{\mathrm{GM}} \mathcal{T}_{g, n}$. Then $f$ induces an automorphism of $\mathcal{C}(S)$.

Proof. It suffices to show that $f(\mathcal{S})=\mathcal{S}$. Then it follows from Lemma 4.5, Lemma 4.6, and Lemma 4.7 that the condition $i(\alpha, \beta)=0, \alpha, \beta \in \mathcal{S}$ is preserved under $f$.

Let $\gamma \in \mathcal{S}$. Denote $[\mathcal{G}]=f([\gamma]) \in \mathcal{P} \mathcal{M F}_{\text {ind }}$. Denote by $\widetilde{\mathcal{G}}$ the unmeasured foliation obtained from $\mathcal{G}$ by forgetting the measure.

First, we observe that the dimension of the space of measures on $\widetilde{\mathcal{G}}$ is one. If not, there exists some other $\mathcal{G}^{\prime} \in \mathcal{P} \mathcal{M} \mathcal{F}_{\text {ind }}$ such that $\mathcal{G}^{\prime}$ is topological equivalent to $\mathcal{G}$, but not projectively equivalent. Denote $[\mathcal{F}]=f^{-1}\left(\left[\mathcal{G}^{\prime}\right]\right) \in \mathcal{P} \mathcal{M} \mathcal{F}_{\text {ind }}$. Since $\mathcal{N}([\mathcal{G}])=\mathcal{N}\left(\left[\mathcal{G}^{\prime}\right]\right)$ and $f^{-1}$ preserves the relation $\bowtie$, we have

$$
\mathcal{N}([\gamma])=\mathcal{N}([\mathcal{F}])
$$

By Lemma 4.7 , we have $[\gamma]=[\mathcal{F}]$, i.e. $f^{-1}\left(\left[\mathcal{G}^{\prime}\right]\right)=f^{-1}([\mathcal{G}])$, which is impossible.

It follows that $\mathcal{G}$ can should one of the following:

(a) $\mathcal{G} \in \mathcal{S}$. In this case, we are done.

(b) $\mathcal{G}$ is uniquely ergodic. This is impossible, since $f$ and $f^{-1}$ preserve $\mathcal{P} \mathcal{M} \mathcal{F}^{U E}$.

(c) The remaining case is that $\mathcal{G}$ is uniquely ergodic on $X_{0}$, which is a proper subsurface of $S$. Let $\beta$ be a boundary component of $X_{0}$. Denote $[\mathcal{F}]=f^{-1}(\beta)$. Then $\mathcal{F}$ is either a simple closed curve or a minimal ergodic component. In both cases, there always exists $\alpha \in \mathcal{S}$ such that $i(\mathcal{F}, \alpha) \neq 0$ and $i(\gamma, \alpha)=0$. Then we have

$$
i(\beta, f(\alpha)) \neq 0, i(\mathcal{G}, f(\alpha))=0 .
$$

Due to our construction, any measured foliation that is intersect with the boundary component $\beta$ should be also intersect with $\mathcal{G}$ (since the measured foliation must cross the collar neighborhood of $\beta$ and $\mathcal{G}$ is filling on the subsurface $X_{0}$ ). This leads to a contraction. 


\section{Proof of Theorem 2}

Let $q \in Q(X)$ be a holomorphic quadratic differential. Define $\mathbf{D}(q)$ be the Teichmüller disk in $\mathcal{T}_{g, n}$ generated by $q$. That is, $\mathbf{D}(q)$ is the image of the holomorphic isometry from the Poincaré disk $\mathbf{D}$ to $\mathcal{T}_{g, n}$, defining by

$$
z \rightarrow X_{z}
$$

where $X_{z}$ is the quasiconformal deformation of $X$ with Beltrami differential $z \bar{q} /|q|$.

Proof of Theorem 2. According to Proposition 4.8, $f$ induces an automorphism of $\mathcal{C}(S)$. By composition with an isometry induced by a mapping class, we may assume that $f=$ id on $\mathcal{S}$.

Choose any pair of simple closed curves $\{\alpha, \beta\}$ that fills the surface. There exists a holomorphic quadratic differential $q$ whose horizontal and vertical foliations are equivalent to $\alpha$ and $\beta$, respectively. Denote by $\mathbf{R}_{1}$ the Teichmüller geodesic directed by $q . \mathbf{R}_{1}$ is contained in the Teichmüller disk $\mathbf{D}_{q}$.

We claim that $f\left(\mathbf{D}_{q}\right)=\mathbf{D}_{q}$.

Let $\tau_{\alpha}$ and $\tau_{\beta}$ denote the Dehn-twists along $\alpha$ and $\beta$, respectively. According to Lemma 3.2 in [17], we know that $\tau_{\alpha}\left(\mathbf{D}_{q}\right)=\mathbf{D}_{q}$ and $\tau_{\beta}\left(\mathbf{D}_{q}\right)=\mathbf{D}_{q}$. Let $\tau=\tau_{\alpha} \circ \tau_{\beta}^{-1}$. From Theorem 7 in [25], $\tau$ is a pseudo-Anosov map. Let $\mathcal{F}$ and $\mathcal{G}$ be the stable and unstable foliations of $\tau$, and let $\mathbf{R}_{2}$ denote the Teichmüller geodesic determined by $\mathcal{F}$ and $\mathcal{G}$. Then $\mathbf{R}_{2} \subset \mathbf{D}_{q}$ and $\tau$ acts on $\mathbf{R}_{2}$ as a translation.

Since $f=$ id on $\mathcal{S}, f\left(\mathbf{R}_{1}\right)$ is also a Teichmüller geodesic terminating at the projective classes of $\alpha$ and $\beta$ (in the Gardiner-Masur boundary). Therefore, $f\left(\mathbf{R}_{1}\right)$ is the Teichmüller geodesic of the holomorphic quadratic differential whose horizontal and vertical foliations are $\alpha$ and $\beta$. Such a Teichmüller geodesic is unique [2, Theorem 5.1] and thus $f\left(\mathbf{R}_{1}\right)=\mathbf{R}_{1}$. Similarly, we have $f\left(\mathbf{R}_{2}\right)=\mathbf{R}_{2}$. This shows that $f\left(\mathbf{R}_{i}\right) \subset \mathbf{D}_{q}, i=1,2$.

For any $X \in \mathbf{D}_{q}$ not on $\mathbf{R}_{1} \cup \mathbf{R}_{2}$, we can take a geodesic $\mathbf{R}_{3}$ passing through $X$ and intersecting with $\mathbf{R}_{1}=f\left(\mathbf{R}_{1}\right)$ and $\mathbf{R}_{2}=f\left(\mathbf{R}_{2}\right)$. This implies that $X \in f\left(\mathbf{D}_{q}\right)$. On the other hand, since the image $f\left(\mathbf{R}_{3}\right)$ is a geodesic, and it intersects $\mathbf{R}_{1}$ and $\mathbf{R}_{2}$, by uniqueness of Teichmüller geodesics, $f\left(\mathbf{R}_{3}\right)$ must contained in $\mathbf{D}_{q}$. Thus $f(X) \in \mathbf{D}_{q}$.

Using Theorem 1 to $\mathbf{D}_{q}$, we have $\left.f\right|_{\mathbf{D}_{q}}=\mathrm{id}$.

According to Proposition 2.2 in [13], we know that the set of geodesics determined by pairs of simple closed curves that fill the surface is dense. This implies that the union of Teichmüller disks which contain such geodesics is dense in $\mathcal{T}_{g, n}$. Hence, we conclude that $f$ is the identity on $\mathcal{T}_{g, n}$.

\section{References}

[1] Fathi, A., F. Laudenbach, and V. Poénaru: Thurston's work on surfaces. - Math. Notes, Princeton Univ. Press, 2013.

[2] Gardiner, F. P., and H. Masur: Extremal length geometry of Teichmüller space. - Complex Var. Elliptic Equ. 16, 1991, 209-237.

[3] HARvey, W. J.: Boundary structure of the modular group. - In: Riemann surfaces and related topics, Proceedings of the 1978 Stony Brook Conference (State Univ. New York, Stony Brook, N.Y., 1978), Ann. of Math. Stud. 97, Princeton Univ. Press, Princeton, N.J., 1981, 245-251.

[4] Hubbard, J., and H. Masur: Quadratic differentials and foliations. - Acta Math. 142, 1979, $221-274$. 
[5] Ivanov, N. V.: Subgroups of Teichmüller modular groups. - Transl. Math. Monogr. 115, Amer. Math. Soc., Providence, RI, 1992.

[6] Ivanov, N. V.: Automorphisms of complexes of curves and of Teichmüller spaces. - Int. Math. Res. Not. IMRN 14, 1997, 51-666.

[7] Ivanov, N. V.: Isometries of Teichmüller spaces from the point of view of Mostow rigidity. In: Topology, Ergodic Theory, Real Algebraic Geometry, Amer. Math. Soc. Transl. Ser. 2, 202, Adv. Math. Sci., 50, Amer. Math. Soc., Providence, RI, 2001, 131-149.

[8] Jeffers, J.: Lost theorems of geometry. - Amer. Math. Monthly 107, 2000, 800-812.

[9] Kerckhoff, S.: The asymptotic geometry of Teichmüller space. - Topology 19, 1980, 23-41.

[10] Korkmaz, M.: Complexes of curves and mapping class groups. - Ph.D. Thesis, Michigan State University, 1996.

[11] Lenzhen, A., and H. Masur: Criteria for the divergence of pairs of Teichmüller geodesics. Geom. Dedicata 144, 2010, 191-210.

[12] Masur, H.: Transitivity properties of the horocyclic and geodesic flows on moduli space. - J. Anal. Math. 39, 1981, 1-10.

[13] Masur, H.: Dense geodesics in moduli space. - In: Riemann surfaces and related topics, 2016, $417-438$.

[14] LI, B., and Y. WAng: Transformations and non-degenerate maps. - Sci. China Ser. A 48, $2005,195-205$.

[15] Li, B., and Y. WANG: Fundamental theorem of geometry without the surjective assumption. - Trans. Amer. Math. Soc. 368, 2016, 6819-6834.

[16] LiU, L., and W. SU: The horofunction compactification of the Teichmüller metric. - In: Handbook of Teichmüller theory. Volume IV, IRMA Lect. Math. Theor. Phys. 19, Eur. Math. Soc., Zürich, 2014, 355-374.

[17] Marden, A., and H. Masur: A foliation of Teichmüller space by twist invariant disks. Math. Scand. 36, 1975, 211-228.

[18] Masur, H.: On a class of geodesics in Teichmüller space. - Ann. of Math. 102, 1975, 205-221.

[19] Masur, H., and M. Wolf: Teichmüller space is not gromov hyperbolic. - Ann. Acad. Sci. Fenn. Ser. A I Math. 20, 1995, 259-267.

[20] Mryachi, H.: Teichmüller rays and the Gardiner-Masur boundary of Teichmüller space. Geom. Dedicata 137, 2008, 113-141.

[21] Mryachi, H.: Teichmüller rays and the Gardiner-Masur boundary of Teichmüller space II. Geom. Dedicata 162, 2013, 283-304.

[22] Miyachi, H.: Extremal length boundary of the Teichmüller space contains non-Busemann points. - Trans. Amer. Math. Soc. 366, 2014, 5409-5430.

[23] Rieffel, M. A.: Group $C^{*}$-algebras as compact quantum metric spaces. - Doc. Math. 7, 2002, 605-651 (electronic).

[24] Royden, H. L.: Automorphisms and isometries of Teichmüller space. - In: Advances in the Theory of Riemann Surfaces, Ann. of Math. Stud. 66, 1970, 369-383.

[25] Thurston, W. P.: On the geometry and dynamics of diffeomorphisms of surfaces. - Bull. Amer. Math. Soc. (N.S.) 19:2, 1988, 417-431.

[26] Walsh, C.: The horoboundary and isometry group of Thurston's Lipschitz metric. - In: Handbook of Teichmüller theory. Volume IV, IRMA Lect. Math. Theor. Phys. 19, Eur. Math. Soc., Zürich, 2014, 327-353.

[27] Walsh, C.: The asymptotic geometry of the Teichmüller metric. - Geom. Dedicata 200:1, $2019,115-152$.

Received 14 January $2019 \bullet$ Accepted 9 September 2019 